\title{
Loop on Skin Modification of Subcuticular Suture Closure of Long Wounds
}

\author{
Deeplaxmi Borle ${ }^{1}$, Senthil Kumar ${ }^{2}$, Anthony Newland ${ }^{3}$ \\ ${ }^{I}$ Post Mch Senior fellow, Institute of Liver and Biliary Sciences \\ New Delhi, India \\ ${ }^{2}$ Additional Professor, Liver transplantation, Institute of Liver and Biliary Sciences, New Delhi, India \\ ${ }^{3}$ Consultant Surgeon, Cross House Hospital, Kilmarnock KA2 OBE
}

Scotland, UK

\begin{abstract}
Closure of long skin wounds using a subcuticular continuous suture may have a few problems such as symptomatic retention of suture resulting in protrusion and pain or irritation, or may become a nidus for infection. We describe a modification of the subcutaneous technique, which allows for removal of the suture soon after healing has been achieved clinically. This is particularly useful for mastectomy wounds.
\end{abstract}

\section{INTRODUCTION}

Approximation of the skin by an absorbable subcuticular (dermal) suture is a common method of wound closure as this gives a good cosmetic result and obviates the need for suture removal. However, even absorbable sutures may be retained in the tissues for longer than is necessary for clinical healing, may contribute to inflammation and infection in contaminated wounds or protrude through the wound causing discomfort ${ }^{1,2}$.Some surgeons therefore, prefer to use a monofilament nonabsorbable subcuticular suture and remove it entirely from the wound milieu in 7 to 10 days. However, removal of a subcuticular suture may pose problems in long wounds such as after a mastectomy or a midline laparotomy where wounds may measure more than 20 centimetres in length. The modification of the standard subcuticular closuretechnique described here consists of introduction of an external loop of suture on the superficial surface of the wound. Thiswhile preserving the advantage of asubcuticular monofilament suture,also makes provision for easy removal of the suture once clinical healing is achieved.

\section{SURGICAL TECHNIQUE}

The technique described is the preferred method of closure of mastectomy wounds by the senior author (ADN), though it could be used for any long wound. A 2/0 prolene suture with retaining beads and collars at each end is used for the subcuticular closure in the standard fashion until about the midpoint of the wound is reached. At this stage the suture is exteriorised to allow placement of a loop on the skin surface (fig.1).

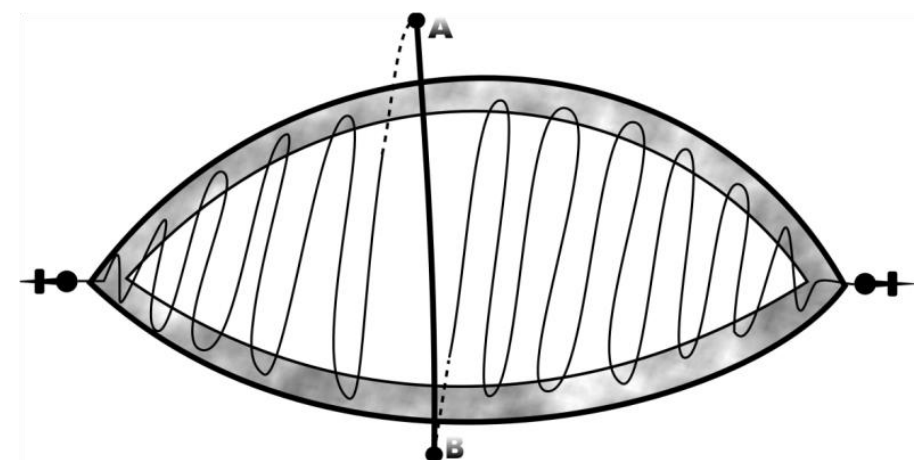

Figure1. Diagramatic representation of the loop on skin modification. The shaded area represents the dermis. In this example the suturing proceeds from the left to the right of the diagram.The external loop is shown by the solid bold line A-B. The thin continuous lines represent the standard subcuticular continuous dermal stitch. 
Starting from the subcuticular position on the inferior flap, the first step in loop formation is the passage of the suture in an inside to outside direction under the superior flap and exiting the superior flap about $2 \mathrm{~cm}$ from the wound edge. The suture is now on the external aspect of the superior flap and has crossed the wound line internally once (first crossing).

The second step is the construction of the external arc of the loop which is achieved by passing the suture in an outside to inside direction on the inferior flap, about $2 \mathrm{~cm}$ from the wound edge crossing the wound line externally (second crossing). The suture is now on the internal aspect of the inferior flap.

The third step involves returning to thesubcuticular mode by taking the next bite on the dermis of the superior flap. The remaining half of the wound is then sutured in the standard subcuticular fashion.

At the time of suture removal the loop is raised and divided as close to the skin, as possible,on both sides, with sterile precautions. The bead and collar at either end is used to draw out half the length of suture from each end.

\section{DISCUSSION}

This technique can be used in any long wound, but is particularly useful in a long wound with flaps such after a mastectomy. The advantage is that there is no residual suture left in the wound after the stipulated period of 7-10 days. Halving the length of suture drawn through the wound eases removal, reduces discomfort to the patient and the chance of suture breakage. There are no specific contraindications for this technique.

\section{REFERENCES}

[1] MehtaPH, DunnKA, BradfieldJF, AustinPE.Contaminated wounds:infectionrates with subcutaneoussutures. Annals of Emergency Medicine 1996; 27 (1): 43-8.

[2] AitkenRJ, AndersonED, GoldstrawS, ChettyU.Subcuticular skin closure following minor breast biopsy: Prolene is superior to polydioxanone (PDS). Journal of the Royal College of Surgeons of Edinburgh 1989; 34 (3): 128-9.

Citation: Deeplaxmi B, Senthil K, Anthony N. Loop on Skin Modification of Subcuticular Suture Closure of Long Wounds. International Journal of Research Studies in Medical and Health Sciences. 2017;2(2):24-25.

Copyright: (C) 2017 Senthil K, et al. This is an open-access article distributed under the terms of the Creative Commons Attribution License, which permits unrestricted use, distribution, and reproduction in any medium, provided the original author and source are credited. 